\title{
FATORES DE RISCO PARA DOENÇAS CRÔNICAS NÃO-TRANSMISSÍVEIS: INQUÉRITO DOMICILIAR NO MƯNICÍPIO DE SÃO PAULO, SP (BRASIL). METODOLOGIA E RESULTADOS PRELIMINARES*
}

\author{
Ricardo A. Rego** \\ Frederico A. N. Berardo** \\ Sérgio S.R. Rodrigues** \\ Zélia M.A. Oliveira** \\ Marcia B. Oliveira*** \\ Cidia Vasconcellos** \\ Lauro V.O. Aventurato** \\ José E.C. Moncau** \\ Luiz R. Ramos**
}

\begin{abstract}
REGO, R.A. et al. Fatores de risco para doenças crônicas não-transmissíveis: inquérito domiciliar no Município de São Paulo, SP (Brasil). Metodologia e resultados preliminares. Rev. Saúde públ., S. Pau10, 24: $277-85,1990$.

RESUMO: As doenças crionicas não-transmissíveis são causa importante de morte no Brasil, principalmente nos grandes centros urbanos. Existem inúmeros fatores de risco relacionados a este tipo de doenças, cuja remoção, ou atenuação, pode contribuir para o declínio da mortalidade. Descreve-se a metodologia do primeiro estudo multicêntrico abrangente realizado na América Latina sobre a questão dos fatores de risco de doenças crônicas não-transmissíveis. No Brasil o estudo foi realizado nos municípios de São Paulo, SP e Porto Alegre, RS. São apresentados resultados preliminares para o Município de São Paulo quanto à prevalência de hipertenção arterial $(22,3 \%)$, tabagismo $(37,9 \%)$, obesidade $(18,0 \%)$, alcoolismo $(7,7 \%)$ e sedentarismo $(69,3 \%)$. Os resultados obtidos são comparados com dados existentes para o Brasil e outros países, e discute-se a relação entre a magnitude dos diversos fatores de risco e a mortalidade por doenças cardiovasculares em São Paulo e alguns países desenvolvidos.
\end{abstract}

DESCRITORES: Doença crônica, epidemiologia. Fatores de risco. Inquéritos de morbidade.

\section{INTRODUÇÃO}

As doenças crônicas não-transmissíveis constituem uma das principais causas de morte nos países desenvolvidos ${ }^{36,40,62}$ e nas grandes cidades brasileiras ${ }^{32}$. Entre essas doenças estão as cardiovasculare ${ }^{36,58,61}$, os cânceres ${ }^{10}$, o diabetes mellitus $^{37}$, as doenças respiratórias crônicas ${ }^{33}$. Tem sido observado aumento na mortalidade por câncer nos Estados Unidos (EUA) ${ }^{2}$ e no Brasil ${ }^{10,64}$.

Entre as doenças cardiovasculares, vem-se percebendo um declínio, nos últimos anos, na mortalidade relativa à doença isquêmica do coração (DIC) ${ }^{35,43,59,65,82}$, e à doença cerebrovascular $41,42,58$, que entretanto não abala a posição deste grupo de doenças como um dos principais componentes da morbimortalidade. Apesar de ainda estar em discussão o que realmente levou a essa queda nas taxas, tem-se atribuído tal fato basicamente a : a) diminuição da exposição aos fatores de risco, como o consumo de cigarros e a ingestão de gorduras ani- mais $5,16,35,44,78,79 ;$ e b) melhorias no diagnóstico, tratamento e cobertura dos serviços de saúde em relação à hipertensão e outras doenças cardiovasculares $34,39,4$ ?

A expressão "fator de risco" refere-se a um conceito que vem ganhando importância crescente no campo das doenças crônicas não-transmissíveis ${ }^{23}$, 63,66. Estas afecções caracterizam-se geralmente por uma etiologia multifatorial e pelo incipiente estado do conhecimento sobre os mecanismos etiológicos e fisiopatológicos que levam ao seu surgimento e desenvolvimento, o que dificulta uma intervenção sistemática e coerente a nível de saúde pública. Entretanto, estudos epidemiológicos têm mostrado consistentemente uma relação entre determinados fatores e determinadas doenças $18,26,45,74,75,77,81$. Além disto, tem-se verificado que a remoção ou reversão da exposição a estes fatores implica na redução da mortalidade e/ou da prevalência $e / o u$ o surgimento mais tardio das patologias em estudo ${ }^{5,40}$. Desta maneira, a intervenção

\footnotetext{
* Realizado com recursos financeiros da Organização Pan-Americana de Saúde e Banco Mundial (Programa Metropolitano de Saúde de São Paulo).

* Seção de Moléstias Degenerativas do Instituto de Saúde - SUDS/SP — Rua Santo Antonio, 590 - $3^{\circ}$ andar 01314 - São Paulo, SP - Brasil.

* * Centro de Vigilância Epidemiológica - SUDS/SP - Av. São Luiz, 99, 70 andar - 01046- São Paulo, SP — Brasil.
} 
sobre as doenças crônicas não-transmissíveis tem como uma de suas vertentes a remoção ou diminuição da exposição a fatores de risco $0^{58,67,71}$, apesar da existência de questionamentos sobre a real efetividade das tentativas de intervenção já realizadas $^{51,57}$.

Tem-se verificado que a distribuição das doenças e dos fatores de risco na população é a expressão de uma realidade socialmente determina$\mathrm{da}^{6,7,8,22,24,31,34,72,76,80,83}$. Neste sentido, impöe-se como de fundamental importância o conhecimento da sua magnitude, quais os mais prevalentes, como se distribuem na população, como base para o planejamento e avaliação de programas de intervenção a nível das doenças crônicas não-transmissíveis.

Apesar desta importância, o volume de informaçôes de que se dispõe sobre a distribuiçãa e comportamento deste tipo de doenças e seus fatores de risco no Brasil ainda é pequeno. Destacam-se como significativos, entre outros, os estudos de $\operatorname{Costa}^{14} \mathrm{e}$ Achutti e Medeiros ${ }^{1}$, no Rio Grande do Sul, e os de Laurenti e Fonseca ${ }^{36}$ e Ribeiro ${ }^{70}$, em São Paulo, sobre hipertensão arterial e doenças cardiovasculares.

A presente pesquisa faz parte de um estudo multicêntrico sobre fatores de risco para doenças crônicas não-transmissíveis desenvolvido em algumas capitais da América Latina (Cidade do México, Havana, Caracas, Santiago-Chile, São Paulo, Porto Alegre), seguindo as diretrizes básicas do Grupo de Saúde do Adulto da Organização Panamericana de Saúde (OPAS), e se constitui no primeiro estudo abrangente sobre este tema realizado no Brasil.

O presente artigo objetiva apresentar a metodologia adotada e alguns resultados preliminares. Análise mais aprofundada dos resultados obtidos será objeto de comunicações posteriores.

\section{METODOLOGIA}

\section{Amostragem}

O estudo foi desenvolvido no Município de São Paulo, em 8 subdistritos (pertencentes ao distrito de Sao Paulo), e 2 distritos. A escolha das regiōes foi realizada de modo intencional. Para os 8 subdistritos, o critério utilizado foi que pertencessem a regiōes socio-economicamente distintas, conforme divisão dos 48 subdistritos em 5 regioes homogêneas proposta por Bussab e Dini ${ }^{11}$. Foram escolhidos 2 distritos com a finalidade de estender este estudo a áreas periféricas e de baixa renda que não estiveram incluídas na divisão em regiões homogêneas mencionadas acima.

Tomaram-se como estratos da amostra regiōes sócio-economicamente semelhantes, da seguinte maneira: A - Subdistritos da Região 1 (Brasilândia e Santo Amaro), B - Subdistritos da
Região 2 (Nossa Senhora do Ó e Jabaquara), C Subdistritos da Região 3 (Butantã e Tucuruvi), D Subdistritos das Regiōes 4 (Santa Cecília) e 5 (Sé), $\mathrm{E}$ - Distritos periféricos (Itaquera e Jaraguá). As regiōes 4 e 5 foram agrupadas no estrato $D$, dada a semelhança sócio-econômica entre ambas.

O estudo abrangeu 100 setores censitários $(20 \mathrm{em}$ cada estrato), nos quais procedeu-se à atualização da listagem dos domicílios existentes, dado que a listagem disponivel havia sido realizada no Censo de 1980.

Em cada um dos estratos sorteou-se uma amostra por conglomerado em dois estágios: a) sorteio dos setores censitários dentro de cada distrito ou subdistrito; b) sorteio dos domicílios dentro de cada setor censitário.

Em função dos objetivos do estudo e da otimização dos recursos disponíveis, determinou-se que o número de domicílios em cada setor censitário seria de 15 , com base na distribuição populacional do Censo de 1980. Devido ao crescimento da população, em cada setor censitário, este número foi corrigido de acordo com a fórmula.

$$
X=\frac{15 \cdot M}{N} \quad \text { onde }
$$

$X=$ número de domicílios a serem sorteados no setor

$\mathrm{N}$ = número de domicílios existentes no setor em 1980

$\mathrm{M}=$ número de domicílios existentes no setor em 1987

(época da atualização citada acima)

Com esta correção, o total de domicilios pesquisados passou a ser de 1.914 .

Este plano amostral foi baseado na experiência de outros estudos já realizados, que utilizaram metodologia semelhante $21,68,84$.

Foi escolhido, em cada domicílio selecionado, um indivíduo entre 15 e 59 anos, utilizando-se para tal o método proposto por Marques e Berquó ${ }^{46}$.

\section{Coleta de Dados}

A todos os indivíduos participantes da amostra foi aplicado um questionário similar ao utilizado nos demais países, ao qual foram acrescentadas questões específicas julgadas importantes para a nossa realidade. Foi realizado um estudo-piloto com a primeira forma do questionário, após o qual chegou-se à redação definitiva. 
O questionário abrangeu os seguintes temas: identificação demográfica e sócio-econômica, percepção de saúde, hipertensão arterial, estresse e apoio social, tabagismo, álcool, saúde da mulher e atividade física.*

Especificamente no estudo realizado em São Paulo, optou-se por uma análise que levasse em conta a distribuição em classes sociais, baseada na sistematização de Barros $^{3}$, e adaptada à proposta de Bronfman e Tuiran'. Os resultados obtidos a partir desta abordagem serão discutidos em trabalhos posteriores.

As medidas de pressão arterial foram realizadas segundo as técnicas padronizadas pela Organização Mundial de Saúde ${ }^{61}$, tendo sido utilizados esfigmomanômetros aneróides devidamente calibrados com aparelhos de coluna de mercúrio e aferidos periodicamente. Duas medidas de pressăo foram feitas, uma no meio e outra no final da entrevista e, para efeito de classificação, foi utilizada a média das duas medições. A pressão sistólica foi considerada na primeira percepção de som e a diastólica na quinta fase de Korotkoff, ou seja, ao desaparecer completamente o som.

As medidas de peso foram efetuadas com balanças portáteis, no final da entrevista, com os indivíduos livres de sapatos e roupas pesadas segundo técnicas padronizadas ${ }^{73}$, registrando-se o peso em kilogramas de tal modo que as frações fossem aproximadas para valores inteiros. As balanças utilizadas foram calibradas com balanças antropométricas devidamente aferidas.

As medidas de altura foram realizadas com trenas portáteis com trava, registrando-se o valor em centímetros, sendo as frações aproximadas para centímetros inteiros.

O procedimento completo durou em torno de 45 a $60 \mathrm{~min}$, na grande maioria dos casos.

$\mathrm{Na}$ seleção dos entrevistadores, optou-se por pessoas sem formação na área médica ou paramédica, com a finalidade de serem evitados vícios de leitura na medida da pressão arterial. Os entrevistadores submeteram-se a treinamento prévio, sob a coordenação dos pesquisadores responsáveis. O treino relativo à pressão arterial envolveu aula informativa a respeito da circulação sanguínea, treino no uso de estetoscópio e esfigmomanômetro, e treino com cronômetro e fita cassete contendo ruídos cardíacos que deveriam ser identificados pelos entrevistadores e registrados em impresso proprio de modo que o início da cronometragem correspondesse à pressão sistólica e o fim da mesma à pressão

* Os interessados poderão obter cópia do questionário junto aos autores do presente artigo. diastólica - método desenvolvido por Rose ${ }^{73}$.

A coleta de dados foi realizada entre março e setembro de 1987 . Houve checagem de $20 \%$ das entrevistas realizadas, sendo que em metade destas o questionário foi aplicado novamente, e nas demais verificou-se apenas a correção do sorteio do entrevistado.

\section{Critérios}

Para a hipertensão utilizaram-se dois critérios: da Organização Mundial da Saúde(OMS) ${ }^{60,61}$, pelo qual se considera hipertenso o indivíduo que apresenta PA diastólica $\geq 95 \mathrm{mmHg}$ e/ou PA sistólica $\geq 160 \mathrm{mmHg}$; do "Joint National Committee" (JNC) ${ }^{27}$, que utiliza os valores de PA diastólica $\geq 90 \mathrm{mmHg}$ e/ou PA sistólica $\geq 140$ $\mathrm{mmHg}$.

A obesidade foi definida ${ }^{85}$ a partir do Indice de Massa Corporal (IMC):

IMC $=\frac{\text { peso }(\mathrm{kg})}{\text { altura }^{2}(\mathrm{~m})}$ Obesidade $=\frac{\text { homens }-\mathrm{IMC} \geq 27,8 \mathrm{~kg} / \mathrm{m}^{2}}{\text { mulheres }-\mathrm{IMC} \geq 27,3 \mathrm{~kg} / \mathrm{m}^{2}}$

Consideraram-se alcoolistas em potencial os indivíduos com teste CAGE positivo $12,19,20,47,48,49,50$.

Foram classificados como sedentários os indivíduos que referiram realizar somente atividade sem esforço físico no tempo de lazer ${ }^{86}$.

Considerou-se como fumantes os indivíduos que relataram fumar diariamente.

\section{Estimação pós-estratificação}

A análise dos resultados mostrou que os dados amostrais obtidos não corresponderam aos que seriam esperados com base no Censo de 1980, para as variáveis sexo e idade, prejudicando assim a representatividade da amostra. Estes desvios foram conseqüência de um erro sistemático da seleção das unidades amostrais no campo. Por uma falha de comunicação com os entrevistadores, as tabelas de sorteio do indivíduo a ser entrevistado em cada domicílio não foram utilizadas de forma totalmente aleatória. Ou seja, estando o entrevistador com vários questionários a serem feitos, ele algumas vezes optou por adotar o questionário que continha a tabela de sorteio que permitisse entrevistar a pessoa presente no domicílio na primeira visita, conseqüentemente levando a uma subestimação da população masculina.

Para correção deste desvio, utilizou-se a técnica de estimação pós-estratificada ${ }^{30}$, que consiste em estratificar a amostra após sua coleta. Neste caso, isto foi feito pelas variáveis sexo e idade (de acordo com os valores do Censo de 1980), conjuntamente dentro de cada estrato, e ponderando as uni- 
dades amostrais de modo que a distribuição dos novos dados refletisse exatamente a distribuição populacional conjunta destas variáveis estratificadoras. Para verificação do procedimento acima, utilizou-se uma variável independente, o estado civil, o que mostrou que os resultados pósestratificação eram adequados.

$\mathrm{Na}$ Tabela $\mathrm{I}$ apresentam-se os resultados obtidos antes e depois da ponderação descrita acima, para as variáveis sexo e idade.

\section{RESULTADOS}

Do total de indivíduos selecionados (1.914) foi possível realizar $1.479(77,2 \%)$ entrevistas, havendo uma perda de $201(10,4 \%)$ por recusa à participação no estudo e de $234(12,2 \%)$ por não haver no domicílio nenhum indivíduo entre 15 e 59 anos completos.

\section{TABELA 1}

Distribuição da população entrevistada segundo sexo e idade. Estudo de fatores de risco para doenças crônicas não-transmissíveis. Município de São Paulo, 1987

\begin{tabular}{lrrrr}
\hline & \multicolumn{3}{c}{ Sexo } \\
Idade (anos) & \multicolumn{2}{c}{ Masculino } & \multicolumn{2}{c}{ Feminino } \\
& $\mathrm{P}$ & $\mathrm{P}$ & $\mathrm{P}$ & $\mathrm{P}$ \\
\hline $15-19$ & 9,6 & 13,5 & 9,6 & 14,0 \\
$20-24$ & 14,5 & 20,7 & 13,2 & 20,0 \\
$25-29$ & 17,3 & 14,3 & 16,8 & 14,5 \\
$30-34$ & 19,3 & 15,7 & 16,9 & 15,4 \\
$35-39$ & 11,8 & 10,2 & 13,1 & 10,6 \\
$40-44$ & 9,2 & 8,4 & 9,4 & 8,0 \\
$45-49$ & 5,6 & 5,0 & 7,9 & 5,9 \\
$50-54$ & 6,8 & 6,8 & 6,6 & 6,4 \\
$55-59$ & 6,0 & 5,3 & 6,4 & 5,2 \\
\hline Total & 100,0 & 100,0 & 100,0 & 100,0 \\
\hline
\end{tabular}

$P=$ percentagem na amostra

$P^{\prime}=$ percentagem ponderada

Os resultados apresentados (Tabela 2) apontam que, dos fatores estudados, a prevalência foi maior no sexo masculino para a hipertensão arterial, tabagismo e alcoolismo. A obesidade e o sedentarismo mostraram-se mais freqüentes no sexo feminino.

A prevalência da hipertensão arterial, segundo os dois critérios utilizados, foi cerca de duas vezes maior para os homens. Quando utilizado o critério do JNC27, para ambos os sexos, as taxas encontradas foram aproximadamente o dobro das obtidas a partir do critério OMS ${ }^{60,61}$.

Para o sexo masculino, a prevalência de tabagismo foi cerca de $40 \%$ maior, e a de alcoolismo em potencial foi aproximadamente quatro vezes maior, em comparação com o sexo feminino.
Para a obesidade e o sedentarismo, a prevalência no sexo feminino foi cerca de $50 \%$ maior do que a encontrada para o sexo masculino.

A comparação com os dados da Pesquisa Nacional por Amostra de Domicnitios (PNAD) de 1987 em relação à população economicamente ativa (Tabela 3) mostrou menor proporção de mulheres economicamente ativas do que as encontradas na população da Grande São Paulo.

\section{TABELA 2}

Prevalência de alguns fatores segundo o sexo. Estudo de fatores de risco de doenças crônicas não-transmissíveis. Município de São Paulo, 1987

\begin{tabular}{lccc}
\hline Fator de Risco & \multicolumn{3}{c}{ Prevalência (em \%) } \\
\hline & Homens & Mulheres & Total \\
\cline { 2 - 4 } $\begin{array}{l}\text { Hipertensos } \\
\text { (critério OMS }\end{array}{ }^{82}$ ) & 15,8 & 7,8 & 11,6 \\
$\begin{array}{l}\text { Hipertensos } \\
\text { (critério JNC }\end{array}$ & 31,0 & 14,4 & 22,3 \\
Obesos & 14,2 & 21,4 & 18,0 \\
Fumantes & 44,6 & 31,9 & 37,9 \\
$\begin{array}{l}\text { Alcoolistas em } \\
\text { potencial }\end{array}$ & 12,6 & 3,3 & 7,7 \\
Sedentários & 57,3 & 80,2 & 69,3 \\
\hline
\end{tabular}

\section{TABELA 3}

Distribuição da população economicamente ativa segundo sexo e idade. Estudo de fatores de risco para doenças crônicas não-transmissíveis (Município de São Paulo) e Pesquisa Nacional por Amostra de Domicílios (Grande São Paulo), 1987

\begin{tabular}{|c|c|c|c|c|}
\hline \multirow{3}{*}{ Idade (anos) } & \multicolumn{4}{|c|}{ População Economicamente Ativa (em \% } \\
\hline & \multicolumn{2}{|c|}{ Homens } & \multicolumn{2}{|c|}{ Mulheres } \\
\hline & PNAD & F R & PNAD & F R \\
\hline $15-19$ & 75,1 & 72,5 & 51,4 & 35,8 \\
\hline $20-24$ & 93,3 & 93,2 & 65,2 & 39,6 \\
\hline $25-29$ & 97,2 & 99,5 & 56,7 & 44,2 \\
\hline $30-39$ & 97,4 & 96,3 & 54,1 & 45,1 \\
\hline $40-49$ & 93,7 & 95,1 & 42,5 & 39,4 \\
\hline $50-59$ & 75,2 & 70,0 & 30,4 & 26,1 \\
\hline
\end{tabular}

PNAD = Pesquisa Nacional por Amostra de Domicilios F R = População do Estudo de Fatores de Risco

\section{DISCUSSÃO E CONCLUSÖES}

Havendo uma sub-representação da população feminina trabalhadora (ver Tabela 3), pode ter acontecido que as entrevistadas neste estudo sejam 
menos aptas (inclusive por doença) para o trabalho do que a população feminina geral, levando conseqüentemente a uma superestimação da prevalência dos diversos fatores de risco no sexo feminino.

Em relação à hipertensão arterial, a prevalência obtida no presente estudo revelou-se maior que a encontrada por Ribeiro69,70 em 1978, para trabalhadores de diversos ramos da indústria, comércio e serviços da Grande São Paulo, que foi de 18,6\% para homens e de $10,4 \%$ para mulheres (critérios: PA diastólica $>90 \mathrm{mmHg}$, população de 15 a 65 anos, padronizado por idade segundo população do presente estudo). Tal diferença pode ser devida ao chamado "healthy worker effect" $25,52,53,87$ que levaria a uma menor prevalência de doenças em populações de trabalhadores em relação à população geral, ou refletir outras diferenças entre as populações estudadas ou, ainda, significar um aumento da prevalência de hipertensão no período, sempre lembrando que o critério de hipertensão de Ribeiro e col. ${ }^{70}$ é mais restrito do que o utilizado por nós. Comparando com o estudo de Costa e Klein ${ }^{15}$, realizado em 1978 no Rio Grande do Sul, que verificou uma prevalência de $13,7 \%$ para homens e $10,0 \%$ para mulheres (critério OMS, população de 20 a 74 anos), pode-se observar uma certa semelhança de resultados, observando-se em São Paulo uma maior diferença entre homens e mulheres. Os dados do "National Health Survey"17 (EUA, 1976-80, população de 18 a 74 anos) mostram prevalência de $32,9 \%$ para homens e $26,8 \%$ para mulheres (critério $\mathrm{JNC})^{27}$, e de $17,4 \%$ para homens e $18,0 \%$ para mulheres (critério OMS) ${ }^{17}$, ou seja, prevalências entre os homens, semelhantes às encontradas no presente estudo, e entre as mulheres cerca de duas vezes maior para as americanas, por ambos os critérios de hipertensão arterial. No Canadá, segundo o nível de escolaridade, a prevalência de hipertensão variou, entre homens, de $21,0 \%$ a $23,0 \%$, e, entre mulheres, de 10,1\% a 15,6\% (PA diastólica $>90 \mathrm{mmHg}$, população de 20 a 69 anos, 1979-81)55.

Quanto ao tabagismo, quando comparado com os dados de 1971 para o Município de São Paulo28,29, que revelaram uma prevalência de $54,8 \%$ de fumantes entre os homens e $20,9 \%$ entre as mulheres (população de 15 a 74 anos, dados padronizados por idade segundo população do presente estudo), parece ter havido uma diminuição da prevalência para o sexo masculino e o inverso para o sexo feminino. Nos EUA, em 1978, a prevalência de tabagismo na população de 17 anos ou mais foi de $37,5 \%$ entre os homens $(51,1 \%$ em 1965$)$ e de $29,6 \%$ entre as mulheres $\left(33,3 \%\right.$ em 1965) ${ }^{78}$. Para o Canadá, verificou-se em 1983 uma prevalência de $37,0 \%$ para homens $(47,2 \%$ em 1975$)$, e $31,5 \%$ para as mulheres (34,4\% em 1975), na população de 20 a 69 anos $^{35}$.

Os dados relativos à obesidade indicam uma prevalência menor em São Paulo do que nos EUA, segundo os dados de Van Itallie ${ }^{85}$, que mostraram prevalência de $24,2 \%$ para os homens e $27,1 \%$ para as mulheres (população de 20 a 74 anos, dados obtidos entre 1976 e 1981, mesmo critério que o adotado no presente estudo). Para a presente população estudada, não existem dados anteriores que permitam uma comparação.

Quanto ao alcoolismo, o único dado populacional existente para São Paulo é o de Capriglione e col. $^{13}$, que verificaram prevalência de teste CAGE positivo de $18 \%$ entre homens e $5 \%$ entre mulheres (populações específicas, 18 a 65 anos de idade, época de coleta de dados não especificada).

Em relação à atividade física, também não se dispõe de dados relativos à população de São Paulo ou outro Estado brasileiro, mas quando se comparam as prevalências de sedentarismo aqui obtidas, entre homens, com aquelas encontradas na GrãBretanha ${ }^{4}$, mesmo levando-se em conta as diferenças metodológicas, pode-se observar uma disparidade entre as taxas, já que no estudo citado a percentagem de homens inativos fisicamente no lazer variou entre 19,8 e $26,1 \%$, conforme o indivíduo pertencesse ao grupo sob intervenção ou ao grupo controle. Nos EUA, estimou-se em 1977 que cerca de metade da população de ambos os sexos fazia exercício físico no tempo de lazer ${ }^{78}$. No Canadá, a prevalência de sedentarismo, em diferentes niveis de escolaridade e em ambos os sexos, não chegou a $20 \%$ para a população de 20 a 69 anos ${ }^{55}$.

Os fatores de risco estudados apresentam taxas relevantes na população do Município de São Paulo. A falta de dados anteriores comparáveis torna impossível uma afirmação segura quanto à tendência evolutiva dos vários fatores de risco em São Paulo, exceto para o tabagismo.

Em relação a comparações com outros países, existem dois grandes problemas: diversidade metodológica, que dificulta ou mesmo impede comparações; e composição etária diferente das populações estudadas, e a freqüente ausência de informação relativa à distribuição dos fatores estudados pelas diversas faixas etárias, inviabilizando a padronização por idade e relativizando as semelhanças e diferenças encontradas.

Feitas estas ressalvas, verifica-se que as prevalências de hipertensão (mulheres) e obesidade (ambos os sexos) parecem ser menores do que as encontradas em alguns países desenvolvidos, como EUA e Canadá, ao contrário do que ocorre com o tabagismo (homens) e o sedentarismo (ambos os sexos). Em relação ao hábito de fumar, parece estar havendo em São Paulo um aumento no sexo feminino, ao contrário do que se observa nos EUA e Canadá, onde o tabagismo vem decrescendo em ambos os sexos.

A comparação com outros países quanto às diferenças entre prevalências de fatores de risco e mortalidade por doenças cardiovasculares pode trazer 
informações interessantes, apesar da dificuldade apresentada, pois este grupo de doenças tem uma história natural de décadas. Deste modo, a mortalidade em um dado período pode estar relacionada com as prevalências de fatores de risco existentes 10 ou 20 anos antes. Como a prevalência tem variado bastante nos países citados ${ }^{55,78}$, as comparaçōes entre elas e a mortalidade por doenças cardiovasculares em diversos países, em um mesmo período, apresentam valor bastante relativo. Ressalte-se que estão relatados no presente estudo apenas alguns fatores de risco dentre todos os relacionados com as doenças em questão.

Lolio e col.43 comparam a mortalidade por doenças cardiovasculares no Município de São Paulo com a encontrada em outros países (população de 40-69 anos, taxas ajustadas por idade, dados relativos a 1980). A mortalidade por DIC em São Paulo, entre os homens, foi cerca de $2 / 3$ da encontrada em países como EUA, Inglaterra e Austrália, e comparável à ençontrada no Canadá, Suécia, Alemanha Ocidental e Israel. Para as mulheres, as taxas em São Paulo foram um pouco menores do que as encontradas para a Inglaterra e EUA, e um pouco maiores que as do Canadá. Para ambos os sexos, a mortalidade por DIC em São Paulo ocupou uma posição intermediária quando comparada com a encontrada nos 27 países industrializados estudados.

No mesmo estudo citado 43 , a mortalidade por doença cerebrovascular em São Paulo foi comparável à encontrada nos países de maior mortalidade (Europa Oriental e Japão), sendo aproximadamente o dobro da encontrada na Inglaterra e o triplo da encontrada nos EUA e Canadá, para ambos os sexos.

Portanto, se as diferenças de mortalidade por DIC parecem compatíveis, a grosso modo, com as diferenças relativas aos fatores de risco estudados, a maior mortalidade por doenças cerebrovasculares em São Paulo parece não seguir o mesmo padrão.
Fazem-se necessários estudos mais aprofundados que indiquem quais fatores de risco ou condiçōes de tratamento são responsáveis pela alta mortalidade por doenças cerebrovasculares em nosso meio, principalmente se levarmos em conta que este grupo de doenças ocupa o terceiro lugar como causa isolada de morte no Município de São Paulo42 e o primeiro lugar no Brasil ${ }^{56}$.

O controle das doenças crônicas nãotransmissiveis requer maior conhecimento sobre os seus fatores de risco. Nesse sentido, seria importante ter-se para o Brasil dados obtidos pelos estudos de coorte, como tem sido feito em outros países. Isto requer uma concentração de recursos humanos e materiais difíceis de serem obtidos no nosso meio, mas a ausência de dados obtidos pelo citado método torna difícil avaliar de maneira mais segura a realidade nesse campo. Além disto, destaca-se como importante o planejamento de um próximo estudo transversal de metodologia semelhante ao atual para acompanhar o que está ocorrendo com os diversos fatores de risco, principalmente no que diz respeito à hipertensão, realização de estudos do mesmo tipo em outras regióes do Brasil e estudos comparativos entre o Brasil e outros países quanto aos fatores de risco e à mortalidade por doenças crônicas não-transmissíveis, especialmente em relação aos dados obtidos com metodologia semelhante em outras capitais latinoamericanas que também participaram deste estudo multicêntrico patrocinado pela OPAS.

\section{AGRADECIMENTOS}

Ao Dr. Luiz Ruiz (Grupo de Saúde do Adulto da OPAS), Dra. Cecilia A. Lolio, Dr. Edmur F. Pastorello, Fernanda M. Zanetta, Dra. Jandira Masur, Dr. José Rosemberg, Dr. Laércio J. Franco, Dra. Luiza S. Heimann, Maria Cristina R. Godoy, Maria Tereza A. Barbosa, Patricia G. A. Ramos, Dr. Wilton $\mathrm{O}$. Bussab, pela colaboração técnica prestada.

REGO, R. A. et al. [Risk factors for non-communicable chronic diseases: a domiciliary enquiry in the City of S. Paulo, Brazil. Methodology and preliminary results]. Rev. Saúde públ., S. Paulo, 24: 277-85, 1990.

ABSTRACT: The non-communicable chronic diseases are important causes of death in Brazil, mainly in the great urban centres. There are various risk factors related to these diseases, whose remotion or attenuation would contribute to a fall in mortality. The methodology of the first comprehensive multicenter study into risk factors of non-communicable chronic diseases carried out in Latin America is explained. In Brazil, this study was carried out in the cities of S. Paulo, SP and Porto Alegre, RS. Preliminary results from the city of S. Paulo as to the prevalence of arterial hypertension $(22.3 \%)$, tabagism $(37.9 \%)$, obesity $(18.0 \%)$, alcoholism $(7.7 \%)$ and sedentarism $(69.3 \%)$ are presented. These results are compared with existing data from Brazil and other countries, and the relationship between various risk factors and the mortality from cardiovascular diseases in S. Paulo and some developed countries is discussed.

KEYWORDS: Chronic disease, epidemiology. Risk factors. Morbidity surveys. 


\section{REFERENCIAS BIBLIOGRÁFICAS}

1. ACHUTTI A. \& MEDEIROS, A. M. B. Hipertensão arterial no Rio Grande do Sul. Bol. Saúde, Porto Alegre, 12(1): 6-54, 1985.

2. BAILAR III, J. C. \& SMITH, E. M. Progress against cancer? New Engl. J. Med., 314: 1226-32, 1986.

3. BARROS, M. B. A. A utilização do conceito de classe social nos perfis epidemiológicos: uma proposta. Rev. Saúde públ., S. Paulo, 20: 269-73, 1986.

4. BAUER, R. L. et al. United Kingdom Heart Disease Prevention Project: 12-year follow up of risk factors. Amer. J. Epidem., 121: 563-9, 1985.

5. BIERMAN, E. L. Atherosclerosis and other forms of arteriosclerosis. In: Isselbacher, K. J. et al., eds. Har. rison's principles of internal medicine. 9th ed. Tokyo, McGraw-Hill Kogakusha, 1980. p. 1156-66.

6. BREILH, J. P. Epidemiologia: economia, medicina y politica. Santo Domingo, SESPAS, 1980.

7. BREILH, J. P. Classe social y desigualdad ante la muerte en Quito. In: Breilh, J. P. et al., ed. Ciudad $y$ muerte infantil. Quito, CEAS, 1983. p. 83-124.

8. BREILH, J. \& GRANDA, E. Investigação da saúde na sociedade: guia pedagógico sobre um novo enfoque no método epidemiológico. São Paulo, Instituto de Saúde/ABRASCO, 1986.

9. BRONFMAN, M. \& TUIRAN, R. La desigualdad ante la muerte: classes sociales y mortalidad en la niñez. Cuad. med. soc., Rosario, (29/30): 53-75, 1984.

10. BRUMINI, R., ed. Câncer no Brasil: dados histopatológicos, 1976-1980. Rio de Janeiro, Instituto Nacional do Câncer, 1982.

11. BUSSAB, W. O. \& DINI, N. P. Pesquisa de emprego e desemprego SEADE/DIEESE: regiōes homogêneas da Grande São Paulo. Rev. Fundação SEADE, S.Paulo, 1(3): 5-11,1985.

12. CAPRIGLIONE, M. J. O questionário CAGE para a detecção da síndrome da dependência do álcool: um estudo com pacientes internados e com uma amostra populacional na cidade de São Paulo. São Paulo, 1984. [Tese de Doutoramento - Escola Paulista de Medicina].

13. CAPRIGLIONE, M. J. et al. Aplicação do questionário CAGE para deteç̧ẫo da síndrome de dependência do álcool em 700 adultos na cidade de São Paulo. Rev. Ass. bras. Psiquiat., 25(7): 50-3, 1985.

14. COSTA, E. A. A cross-sectional survey of blood pressure in Rio Grande do Sul, Brazil. London, 1981. [Tese de Doutoramento - London University].

15. COSTA, E. A. \& KLEIN, C. H. Meio urbano e doenças cardiovasculares. Cad. Saúde públ., Rio de Janeiro, 1: 305-12, 1985.
16. DOLL, R. \& PETO, R. Mortality in relation in smoking: 20 years, obervation on male British doctors. Brit. med. J., 2: 1525-36, 1976.

17. DRIZD, A. L. et al. Blood pressure levels in persons $18-74$ years of age in 1976-80, and trends in blood pressure from 1960 to 1980 in the United States. Vital Hlth Statist. Ser. 11 (234) 1986.

18. EDWARDS, G., et al., ed. Alcohol related disabilities. Geneva, 1977. (WHO-Offset Publication, 32).

19. EWING, J. A. Detecting alcoholism. The CAGE questionnaire. J. Amer. med. Ass., 252: 1905-7, 1984.

20. EWING, J. A. \& ROUSE, B. A. Identifying the hidden alcoholic. [Apresentado no 29th International Congress on Alcoholism and Drug Dependence, Sidney, Australia, 1970].

21. FUNDAÇÃo IBGE. Metodologia da Pesquisa Nacional por Amostra de Domicilios (PNAD) na década de 70. Rio de Janeiro, 1981.

22. GARFIELD, J. Alienated labor, stress and coronary disease. Int. J. Hlth Serv., 10: 551-61, 1980.

23. GRUNDY, P. F. A rational approach to the "at risk" concept. Lancet, 2: 1489, 1973.

24. HENRY, J. P. The relation of social to biological processes in disease. Soc. Sci. Med., 16: 369-80, 1982.

25. HERNBERG, S. Work related diseases: some problems in study design. Scand. J. Work environ. Hlth, 10: $367-72,1984$

26. HOPKDNS, P. N. \& WILLIAMS, R. R. A survey of 276 suggested coronary risk factors. Atherosclerosis, 40: $1-52,1981$.

27. JONNT NATIONAL COMMITTEE DETECTION EVALUATION AND TREATMENT OF HIGH BLOOD PRESSURE. The 1984 Report of the Joint National Committee on Detection, Evaluation, and Treatment of High Blood Pressure. Arch. intern. Med., 144: 1045-57, 1984.

28. JOLY, D. J. Encuesta sobre las caracteristicas del habito de fumar en America Latina. Washington, DC, Organizacion Panamericana de la Salud, 1977. (OPAS - Publicacion Cientifica, 337).

29. JOLY, D. J. El habito de fumar cigarrillos en la America Latina: una encuesta en ocho ciudades. Bol. Ofic. sanit. panamer; 79: 93-111, 1975.

30. KISH, L. Muestreo de encuestas. Mexico, Ed. Trillas, 1972.

31. LAURELI, A.C. A saúde-doença como processo social. In: Nunes, E. D., ed. Medicina social: aspectos históricos e teóricos. São Paulo, Global, 1983. p. 133-58. 
32. LAURENTI, R. O problema das doenças crônicas e degenerativas e dos acidentes nas áreas urbanizadas da América Latina. Rev. Saúde públ., S. Paulo, 9: 23948, 1975.

33. LAURENTI, R. Doenças respiratórias como causa de morte no Município de São Paulo, SP (Brasil). Rev. Saúde públ., S. Paulo, 15: 353-63, 1981.

34. LAURENTI, R. Mortalidade por hipertensão como causa básica. Ciênc. e Cult., 35: 1637-42, 1983.

35. LAURENTI, R. O declínio das doenças cardiovasculares como causa de morte. Rev. Saúde públ., S. Paulo, 20: $339-40,1986$

36. LAURENTI, R. \& FONSECA, L. A. M. A mortalidade por doenças cardiovasculares no Município de São Paulo em um periodo de 30 anos (1940-1969). Arq. bras. Cardiol., 29: 85-8, 1976.

37. LAURENTI, R. et al. Mortalidade por diabetes mellitus no Município de São Paulo (Brasil). Evolução em um periodo de 79 anos (1900-1978) e análise de alguns aspectos sobre associação de causas. Rev. Saúde públ., S. Paulo, 16: 77-91, 1982.

38. LEAVELL, H. R. \& CLARK, E. G. Medicina preventiva. São Paulo, McGraw-Hill do Brasil, 1978.

39. LEVY, R. I. Progress toward prevention of cardiovascular disease. Circulation, 60: 1555-9, 1979.

40. LEVY, R. I. \& FEINLEIB. M. Fatores de risco para doença arterial coronariana e seu controle. In: Braunwald, E., ed. Tratado de medicina cardiovascular. São Paulo, Ed. Roca, 1987.v. 2, p. 1176-205.

41. LEVY, R. I. \& MOSKOWITZ, J. Cardiovascular research: decades of progress, a decade of promise. Science, 217: 121-9, 1982.

42. LOLIO, C. A. \& LAURENTI, R. Tendência da mortalidade por doenças cerebrovasculares em adultos majores de $\mathbf{2 0}$ anos de idade no Município de São Paulo (Brasil), 1950 a 1981. Rev. Saúde públ., S. Paulo, 20: 343.6, 1986.

43. LOLIO, C. A. et al. Decline in cardiovascular disease mortality in the city of São Paulo, Brazil, 1970 to 1983. Rev. Saúde públ., S. Paulo, 20: 454-64, 1986.

44. LUEPKER, R. V. et al. Population risk of cardiovascular disease: The Minnesota Heart Survey. $J$. chron.Dis., 38: 671-82, 1985.

45. MARMOT, M. G. Stress and cultural variations in heart disease. J. Psychosom. Res., 27: 377-84, 1983.

46. MARQUES, R. M. \& BERQUO, E. Seleção da unidade de informação em estudos de tipo "survey": um método para a construção das tabelas de sorteio. Rev. bras. Estat., 37 (145): 81-92, 1976.

47. MASUR, J. C. \& MONTEIRO, M. G. Validation of the "CAGE" alcoholism screening test in a Brazilian inpatient hospital setting. Braz. J. med. biol. Res., 16: $215-8$, 1983.
48. MASUR, J. C. et al. Hipertensão e alcoolismo. Rev. Ass. med. bras., 26(3): 86-8, 1980.

49. MASUR, J. C. et al. Detecção precoce do alcoolismo em clínica médica através do questionário CAGE: Utilidade e limitaçōes. J. bras. Psiquiat, 34: 31-4, 1985.

50. MAYFIELD, D. G. et al. The CAGE questionnaire: validation of a new alcoholism instrument. Amer. $J$. Psychiat., 131: 1121-3, 1974.

51. MCCORMICK, J. \& SKRABANEK, P. Coronary heart disease is not preventable by population interventions. Lancet, 2: 839-41, 1988.

52. MCMICHAEL, A. J. et al. An epidemiologic study of mortality within a cohort of rubberworkers, 196472. J. occup. Med., 16: 458-64, 1974.

53. MCMICHAEL, A. J. Standardized mortality ratios and the healthy worker effect: scratching beneath the surface. J. occup. Med., 18: 165-8, 1976.

54. MCQUEEN, D. V. \& SIEGRIST, J. Social factors in the etiology of chronic disease: an overview. Soc. Sci. Med., 16: 353-67, 1982.

55. MILLAR, W. J. \& WIGLE, D. T. Socioeconomic disparities in risc factors for cardiovascular disease. Canad. med. Ass. J., 134: 127-32, 1986.

56. MINISTÉRIO DA SAUDE. Estatisticas de mortalidade: Brasil, 1985. Brasília, 1988.

57. OLIVER, M. F. Prevention of coronary heart disease - propaganda, promises, problems and prospects. Circulation, 73: 1-9. 1986

58. ORGANIZAC̄ÃO MUNDIAL DA SAÚDE. Primary prevention of coronary heart disease, Anacapri, 1984. (WHO - Euro Reports and Studies, 98).

59. ORGANIZAÇÃO MUNDIAL DA SAUDE. Mortality from ischaemic hean disease in industrialized countries. Wkly. Epidem. Rec., 62: 233-6, 1987.

60. ORGANIZAÇÃO MUNDIAL DA SAÚDE. Expert Committee on Cardiovascular Diseases and Hypertension, Geneva, 1958. Hypertension and coronary heart disease: clessification and criteria for epidemiological studies; first report. Geneva, 1959. (Technical Report Series, 168).

61. ORGANIZAÇÃO MUNDIAL DA SAÚDE. Comite de Expertos en Hypertension Arterial, Ginebra, 1978. Informe. Ginebra, 1978. (Serie de Informes Tecnicos, 628).

62. ORGANIZAÇÃO MUNDIAL DA SAÚDE. Expert Committee on Community Prevention and Control of Cardiovascular Diseases, Geneva, 1984. Report. Geneva, 1986. (Technical Report Series, 732).

63. ORGANIZAÇÃO PAN-AMERICANA DE SAÚDE. Enfer. medades cronicas del adulto: estudio de factores de riesgo individuales y poblaciones susceptibles de intervencion. Plan de desarollo. Programa de Salud del Adulto. Washington, D. C., 1985. 
64. PASTORELO, E. F. Mortalidade por câncer no Distrito de São Paulo, 1962/1963 e 1974/1975. São Paulo, 1980. [Dissertação de Mestrado - Faculdade de Saúde Pública da USP].

65. PISA, Z. \& UEMURA, K. Trends of mortality from ischemic heart disease and other cardiovascular diseases in 27 countries, 1968-1977. Wld Hlth Statist. Quart., 35: 11-7, 1982.

66. PLAUT, R. Analisis de riesgo: alcance y limitaciones para el administrador de salud. Bol. Ofic. sanit. panamer., 96: 296-306, 1984.

67. PUSKA, P. et al. Ten years of the North Karelia Project: results with community-based prevention of coronary heart disease. Scand. J. soc. Med., 11: $65-8,1983$.

68. RAMOS, L. R. Growing old in São Paulo, Brazil: assesment of health status and social support of elderly people from different socio-economic strata living in the community. London, 1987. [PhD Dissertation - London School of Hygiene and Tropical Medicine, London University].

69. RIBEIRO, A. B. \& RIBEIRO, M. B. D. Hypertension in underdeveloped countries. Drugs, 31 (Suppl. 4): 23-8, 1986.

70. RIBEIRO, M. B. D. et al. Hypertension and economic activities in São Paulo, Brazil. Hypertension, 3 (6 pt 2): ПI-233-7, 1981.

71. ROSE, G. Strategy of prevention: lessons from cardiovascular disease. Brit. med. J., 282: 1847-51, 1981.

72. ROSE, G. A. \& MARMOT, M. G. Social class and coronary heart disease. Brit. Heart J., 45: 13-9, 1981.

73. ROSE, G. A. et al. Metodos de encuesta sobre enfermedades cardiovasculares. 2." ed. Ginebra, Organización Mundial de la Salud, 1982. (OMS-Serie de Monografias, 56).

74. ROSEMBERG, J. Tabagismo: sério problema de saúde pública. São Paulo, ALMED/Ed. USP, 1981.

75. ROSEMBERG, J. Riscos a que se expōem os fumantes passivos: direitos dos nāo-fumantes. Rev. Ass. med. bras., 31 (1/2): 7-12, 1985.
76. RUSSEK, H. I. Stress, tobacco and coronary disease in North American professional groups: survey of 12,000 men in 14 occupational groups. J. Amer. med. Ass., 192: 89-94, 1965.

77. STAMLER, J. Cardiologia preventiva. Barcelona, Ed. Científico-Médica, 1970.

78. STAMLER, J. Primary prevention of coronary heart disease: the last 20 years. Amer. J. Cardiol. 47: 722-35, 1981.

79. STAMLER, J. Coronary heart disease: doing the "right things". New Engl. J. Med., 312: 1053-5, 1985.

80. SYME, S. L. \& BERKMAN, L. F. Social class, susceptibility and sickness. Amer. J. Epidem., 104: 1003-6, 1976.

81. THELLE, D. S. et al. The Tromso Heart Study: methods and main results of the cross sectional study. Acto med. scand., 200: 107-18, 1976.

82. THOM, T. J. et al. Trends in total mortality and mortality from heart disease in 26 countries from 1950 to 1978. Int. J. Epidem., 14: 510-28, 1985.

83. TIMIO, M. Classes sociales y enfermedad. $2^{\mathbf{a}}$ ed. Mexico, Nueva Imagen, 1980.

84. U. S. BUREAU OF THE CENSUS. Atlantida: a case study in household serveys-Unit IV - Sample Desing. Washington, DC, 1966. (Series ISPO 1, No 1E).

85. VAN ITALLIE, T. B. Health implications of overweight and obesity in the United States. Ann. intern. Med., 103: 983-8, 1985.

86. WASHBURN, R. A. \& MONTOYE, H. J. The assessment of physical activity by questionnaire. Amer. J. Epidem., 123: 563-76, 1986.

87. WEED, D. L. Historical roots of the healthy worker effect. J. occup. Med., 28: 343-7, 1986.

Recebido para publicação em 24/10/1989 Reapresentado em 26/3/1990 Aprovado para publicação em 28/3/1990 